ИЗВЕСТИЯ АКАДЕМИИ НАУК ЭСТОНСКОИ ССР. ФИЗИКА * МАТЕМАТИКА

PROCEEDINGS OF THE ACADEMY OF SCIENCES OF THE ESTONIAN SSR. PHYSICS * MATHEMATICS

$1987,36,2$

\title{
К МЕТОДАМ С АППРОКСИМАЦИЕЙ ОБОБЩЕННОГО ПСЕВДООБРАТНОГО ОПЕРАТОРА
}

\author{
(Представил Н. Алумяэ)
}

Понятие псевдообратного оператора позволяет укладывать в единые рамки различные итерационные методы, в кратком и сжатом виде сформулировать научные результаты. Для вычисления обобщенного решения нелинейного операторного уравнения в терминах обобщенной псевдоннверсии используется одно общее семейство итерационных методов, включающее в себя как частные случаи методы Гаусса-Ньютона, БенИзраэля, Левенберга-Марквардта, а также методы с последовательной аппроксимацией обобщенной псевдоинверсии.

1. Пусть $F(x)$ - дифференцируемый оператор из одного гильбертова пространства $H_{1}$ в другое $H_{2}$, и оператор $F^{\prime}(x)$ имеет замкнутый образ $R(x)=R\left(F^{\prime}(x)\right)$, причем его нулевое подпространство может быть нетривиальным. В силу замкнутости образа $R(x)$ существует равномерно ограниченный псевдообратный оператор $\left[F^{\prime}(x)\right]^{+}$. Положим

$$
\Phi(x)=\left[F^{\prime}(x) U^{-1}\right]^{+}, \quad \Psi(x)=\Phi^{+}(x)=F^{\prime}(x) U^{-1},
$$

где $U$ - произвольный линейный несингулярный оператор из $H_{1}$ в себя. Через $P_{R(x)}=P_{R(\Psi(x))}\left(P_{k}=P_{R\left(\Psi\left(x_{k}\right)\right)}\right)$ обозначим оператор ортогонального проектирования $H_{2}$ на $R(\Psi(x))$.

Для решения уравнения

$$
\left[F^{\prime}(x)\right]^{*} F(x)=0
$$

рассмотрим методы вида

$$
x_{k+1}=x_{k}-\varepsilon_{k} A_{k} F\left(x_{k}\right),
$$

где $\varepsilon_{k}-$ релаксационный (доминирующий) параметр $0<\varepsilon_{k} \leqslant 1, A_{k}-$ линейный оператор из $H_{2}$ в $H_{1}$, аппроксимирующий оператор $U^{-1}\left(F^{\prime}\left(x_{k}\right) U^{-1}\right)^{+}$. Согласно терминологии [ $\left.{ }^{1}\right]$, оператор $U^{-1}\left(F^{\prime}(x) U^{-1}\right)^{+}$ можно назвать $I U$-взвешенным псевдообратным оператором. Введение несингулярного оператора $U$ не имеет принципиального значения, а обусловлено удобством при рассмотрении некоторых проблем в вычислительной практике. В общем случае в качестве $U$ допускается брать и сингулярный линейный оператор [ $\left.{ }^{1}\right]$.

Пусть далее $C, \bar{C}, \bar{L}_{0}, L_{1}, N, N^{\prime}, N_{0}, L$ и $\lambda$ - означают некоторые положительные константы. Предположим, что $A_{k}$ является аппроксимацией для $\left[F^{\prime}\left(x_{k}\right) U^{-1}\right]^{+}$, т. е. $A_{k}=U A_{k}$, и справедливы следующие соотношения

$$
\bar{A}_{k}=\bar{A}_{k} P_{k}, \quad\left\|\bar{A}_{k}\right\| \leqslant \bar{\lambda}_{k} \leqslant \bar{\lambda}<\infty, \quad\left\|A_{k}\right\| \leqslant \lambda_{k} \leqslant \lambda<\infty .
$$

С использованием результатов из $\left[{ }^{2}\right]$ нетрудно доказать следующую лемму.

Л е м м. Пусть $F(x)$ - Липшиц непрерывен и Ф $(x)$ - равномерно ограниченный оператор в рассматриваемой области $S$, т. е. 


$$
\begin{array}{ll}
\left\|F^{\prime}(x)-F^{\prime}(y)\right\| \leqslant L_{1}\|x-y\|, & \forall x, y \in S, \\
\|\Phi(x)\| \leqslant \bar{C}, & \forall x \in S,
\end{array}
$$

тогда

$$
\left\|P_{R(x)}-P_{R(y)}\right\| \leqslant \bar{L}_{0}, \quad \forall x, y \in S, \quad \text { где } \quad \bar{L}_{0}=\bar{C} L_{1}\left\|U^{-1}\right\| .
$$

Из леммы вытекает, что существуют постоянные $N^{\prime}$ и $N$ такие, что

$$
\begin{gathered}
\left\|\left(P_{R(y)}-P_{R(y)} P_{R(x)}\right) F(x)\right\| \leqslant N^{\prime}\|x-y\|, \\
\left\|\left(P_{k+1}-P_{k+1} P_{k}\right) F\left(x_{k}\right)\right\| \leqslant \varepsilon_{k} N\left\|P_{k} F\left(x_{k}\right)\right\| .
\end{gathered}
$$

Путем ввода последовательности $\left\{\bar{\gamma}_{k}\right\}$, определяемой по условию

$$
\| P_{k}-\Psi\left(x_{(k)} \bar{A}_{k} \| \leqslant \bar{\gamma}_{k}, \quad k=0,1, \ldots,\right.
$$

получается

Т е о р ем а 1. Пусть $x_{0} \in H_{1}, S=\left\{x \in H_{1}:\left\|x-x_{0}\right\| \leqslant \mathrm{e}\right\}$ и выполнены условия:

$1^{\circ}$ оператор $F(x)$ дифференцируем (по Фреше);

$2^{\circ}$ производная $F^{\prime}(x)$ удовлетворяет условию Липшица

$$
\left\|F^{\prime}(x)-F^{\prime}(y)\right\| \leqslant L_{1}(\|x-y\| ;
$$

$3^{\circ} \delta=\delta_{0}<1$ ( $\delta_{0}$ определяется ниже в разных случаях по разному $)$; $4^{\circ} 0<\varepsilon_{0} \leqslant \varepsilon_{k-1} \leqslant \varepsilon_{k}=\min \left\{1, \varepsilon_{k-1} \delta_{k-1}^{-1 / 2}\right\}, \quad\left\|P_{k+1} F\left(x_{k+1}\right)\right\| \leqslant \delta_{k}\left\|P_{k} F\left(x_{k}\right)\right\|$.

A. Тогда, если существует постоянное $\bar{C}$ такое, что $\|\Phi(x)\| \leqslant \bar{C}$, $\bar{\gamma}_{k} \leqslant \bar{\gamma}_{0}<1, r_{1}=\lambda\left\|P_{0} F\left(x_{0}\right)\right\| /(1-\delta) \leqslant 0, \quad$ то уравнение $\left[F^{\prime}(x)\right]^{*} F(x)=0$ имеет в $S$ решение $x^{*}, \kappa$ которому сходится последовательность (3), причем $\left\|x^{*}-x_{0}\right\| \leqslant r_{1}, \delta_{k} \leqslant \delta u$

$$
\left\|x_{k}-x^{*}\right\| \leqslant r_{1} \delta^{k},
$$

где $\delta=\delta_{0}=1-\varepsilon_{0}+\varepsilon_{0}\left(\bar{\gamma}_{0}+N\right)+\frac{1}{2} \varepsilon_{0}^{2} \lambda^{2} L_{1}\left\|P_{0} F\left(x_{0}\right)\right\|$.

Б. Если $P_{R(x)}$ не зависит от х и $\bar{\gamma} \geqslant \bar{\gamma}_{0} \geqslant \ldots \geqslant \bar{\gamma}_{k} \geqslant \ldots \geqslant 0, \bar{\gamma}_{k} \rightarrow 0$ при $k \rightarrow \infty$, то последовательность $\left\{x_{k}\right\}$ сходится со сверхлинейной скоростью, причем $\left\|x^{*}-x_{0}\right\| \leqslant r_{1}$

$$
\left\|x_{k}-x^{*}\right\| \leqslant r_{1} \prod_{i=0}^{k-1} \delta_{i}
$$

где $\delta_{i}=1-\varepsilon_{i}+\varepsilon_{i} \bar{\gamma}_{i}+\frac{1}{2} \varepsilon_{i}^{2} \lambda^{2} L_{1}\left\|P_{i} F\left(x_{i}\right)\right\| \quad$ при $i \rightarrow \infty$. Это доказательство повторяет с необходимыми изменениями доказательство теоремы из $\left[{ }^{3}\right]$. В качестве примера этих изменений выведем здесь следующее соотношение:

$$
\begin{gathered}
\left\|\left[F^{\prime}(x)\right]^{*} F(x)\right\| \leqslant\|H\|\left\|H^{-1}\left[F^{\prime}(x)\right]^{*} F(x)\right\| \leqslant\|H\|\left\|U^{-1}\left(F^{\prime}(x) U^{-1}\right)^{*} F(x)\right\| \leqslant \\
\leqslant\|H\|\left\|U^{-1}\left(F^{\prime}(x) U^{-1}\right)^{*} \Psi(x) \Phi(x) F(x)\right\| \leqslant \\
\leqslant\|H\|\left\|U^{-1}\left(F^{\prime}(x) U^{-1}\right)^{*}\right\|\left\|P_{R(x)} F(x)\right\| .
\end{gathered}
$$

Отсюда в силу непрерывности оператора $P_{R(x)} F(x)$ следует, что решение уравнения $P_{R(x)} F(x)=0$ является также и решением уравнения (2).

Отметим еще, что в $\left[{ }^{3-6}\right]$ при выведении оценки для квадратичной скорости последовательности (3) были использованы следующие условия:

$$
\begin{gathered}
\left\|P_{R(x)}-P_{R(y)}\right\| \leqslant L\|x-y\|, \\
\left\|P_{R(y)} P_{R(x)} P_{R(y)}-P_{R(y)} P_{R(x)}\right\| \leqslant N_{1}\|x-y\|\|x-y\|^{2} .
\end{gathered}
$$


Из выполнимости условий (8) и (9) следует, что в этом случае $P_{R(x)}$ не зависит от $x$, и поэтому условия сходимости для метода (3) можно записать в более простом виде.

В случае $R(x) \supseteq R(y)$ или в случае, когда $P_{R(x)}$ и $P_{R(y)}$ перестановочны, условие (9) выполняется также при $N=0$.

2. Семейство методов (3) включает в себя как частные случаи релаксационные методы типа Левенберга-Марквардта

$$
x_{k+1}=x_{k}-\varepsilon_{k} D_{k}\left[F^{\prime}\left(x_{k}\right)\right]^{*} F\left(x_{k}\right),
$$

где $D_{k}$ - некоторая аппроксимация для $M\left(x_{k}, \alpha_{k}\right)=\left[F^{\prime}\left(x_{k}\right)\right]^{*} F^{\prime}\left(x_{k}\right)+$ $+\alpha_{k} H, \alpha_{k}>0, H=U^{*} U$ и $A_{k}=D_{k}\left[F^{\prime}\left(x_{k}\right)\right]^{*}$, так как $\lim _{\alpha_{k} \rightarrow 0} M_{k}^{-1}\left[F^{\prime}\left(x_{k}\right)\right]^{*}=$ $=U^{-1}\left[F^{\prime}\left(x_{k}\right) U^{-1}\right]^{+}\left[{ }^{1}\right]$.

Пусть $\omega_{k}=\mu_{k} / \alpha_{k}$ и $\mu_{k}$ и $K$ некоторые постоянные, удовлетворяющие неравенствам

$$
\left\|I-M_{k} D_{k}\right\| \leqslant \mu_{k}<1, \quad\left\|F^{\prime}(x)\right\| \leqslant K,
$$

тогда имеет место

Сл едс т в и е. Пусть $x_{0} \in H_{1}, \quad S=\left\{x \in H_{1}:\left\|x-x_{0}\right\| \leqslant \varrho\right\}$ ина $S$ выполнены условия $1^{\circ}-4^{\circ}$ теоремы 1 .

Eсли $\|\Phi(x)\| \leqslant \bar{C}, \quad \alpha_{k} \leqslant \alpha_{0}, \quad \omega_{k} \leqslant \omega_{0} \quad\left(\alpha_{0}, \omega_{0}<\infty\right), \quad r_{1}=\lambda\left\|P_{0} F\left(x_{0}\right)\right\| /$ $/(1-\delta) \geqslant 0$, где $\delta=\delta_{0}=1-\varepsilon_{0}+\varepsilon_{0}\left[N+K\left\|U^{-1}\right\|\left(\alpha_{0} \bar{C}^{3}+\omega_{0} K\|U\| H^{-1} \|\right)\right]+$ $+\frac{1}{2} \varepsilon_{0} \lambda^{2} L_{1}\left\|P_{0} F\left(x_{0}\right)\right\|$, то уравнение (2) имеет в $S$ решение $\mathrm{x}^{*}, \kappa$ которому сходится последовательность (10), причем

$$
\left\|x^{*}-x_{0}\right\| \leqslant r_{1} \quad \text { и } \quad\left\|x_{k}-x^{*}\right\| \leqslant r_{1} \delta^{k} .
$$

Ниже рассмотрим некоторые способы построения оператора $D_{k}$. Пусть $D_{k}{ }^{(0)}-$ некоторая аппроксимация для $M_{k}^{-1}=\left[M\left(x_{k}, \alpha_{k}\right)\right]^{-1}$ такая, что $\left\|T_{k}\right\|<1$, где $T_{k}=I-M_{k} D_{k}{ }^{(0)}$ и $I-$ тождественный оператор.

Для построения $D_{k}$ можно использовать формулу

$$
D_{k} \equiv D_{k}^{(q)}=D_{k}^{(0)}\left[I+T_{k}+\ldots+T_{k}^{q}\right], \quad q \geqslant 0 .
$$

В частности, в качестве $D_{k}{ }^{(0)}$ можно принять $D_{k}{ }^{(0)}=a_{k} I$, где $0<a_{k}<2 /\left(K^{2}+\alpha\|H\|\right)$. В последнем случае справедливы соотношения $\left\|I-M_{k} D_{k}\right\| \leqslant v_{k}^{q}, \quad\left\|A_{k}\right\| \leqslant \lambda_{q}, \quad$ где $\quad\left\|I-a_{k} M_{k}\right\| \leqslant v_{k} \quad$ и $\quad \lambda_{q}=$ $=\min \left\{\frac{2}{K}\left(1+v+\ldots+v^{q}\right)\right.$, и $\left.\left\|U^{-1}\right\|\left(\bar{C}+\alpha_{0} \bar{C}^{3}+\omega_{0} K\|U\|\left\|H^{-1}\right\|\right)\right\}$. Итак, можем положить $\mu_{0}=v^{q}$ и $\lambda=\lambda_{q}$.

При $q=1$ один эффективный способ такого типа для вычисления $D_{k}$ описан в $\left[{ }^{7}\right]$.

В общем случае для повышения точности аппроксимации оператора $M_{k}{ }^{-1}$ можно воспользоваться многочленами Чебышева. Образуем линейную комбинацию

$$
S_{h}^{(q)} \equiv \sum_{i=0}^{q} a_{q, i} D_{h}^{(i)}, \quad q \geqslant 2
$$

где коэффициенты многочлена Чебышева $Q_{q}(x)=\sum_{i=0}^{q} a_{q, i} x^{i} \quad$ удовлетворяют условию $\sum_{i=0}^{q} a_{q, i}=1$. Тогда

$$
\left\|I-M_{k} S \frac{q}{k}\right\| \leqslant v / \eta_{q} \leqslant 2 v^{q+1} /\left(1+\sqrt{1-v^{2}}\right)^{q},
$$




$$
\begin{gathered}
\lambda=\lambda_{q}=\min \left\{\frac{2}{K}\left(1+v+\ldots+v^{q}\right) \sum_{i=0}^{q}\left|a_{q, i}\right|, \ldots\right. \\
\left.\ldots\left\|U^{-1}\right\|\left(\bar{C}+\alpha \bar{C}^{3}+\frac{v \eta_{q}}{2} K\|U\|\left\|H^{-1}\right\|\right)\right\}
\end{gathered}
$$

где $v=\sup \left\{v_{k}\right\}$ и $\eta_{q}=1 /\left|Q_{q}(1 / v)\right|$.

Для построения $A_{k}$ в случае $U=I$ аналогичные результаты, основанные на разложении псевдообратного оператора в ряд Неймана, получены в [ $\left.{ }^{4}\right]$.

В конечномерном случае $\left(F: R^{n} \rightarrow R^{m}\right)$ при вычислении очередного приближения $x_{k}$ для решения $x^{*}$ по формулам $(10),(11)$ и $(10)$, (12) основной операцией является переумножение матрицы на вектор, которая требует $O(m n)$ умножений, и поэтому такой подход экономен для решения задач большой размерности. В случае задач с редким якобианом число арифметических операций, требуемое для совершения одного итерационного шага, значительно сокращается.

\section{ЛИТЕРА Т У Р А}

1. Elden, L. A Note on Weighted Pseudoinverses with Application to the Regularization of Fredholm Integral Equations of the First Kind. Report LitH-MAT-R-1975-11. Linköping University (Sweden), Department of Mathematics, 1975.

2. Красносельский М. А., Вайникко І. М., Забрейко П. П., Рутщкий Я. Б., Стеценко В.Я. Приближенное решение операторных уравненнй. М., «Наука», 1969.

3. Вааряанн О., Ломп М. Изв. АН ЭССР. Фнз. Матем., 31, № 4, 410-417 (1982).

4. Ваарманн О. Изв. АН ЭССР. Физ. Матем., 20, № 4, 386-394 (1971).

5. Vaarmann, O. ENSV TA Toim. Füüs. Matem., 29. № 3, 233-240 (1980).

6. Vaarmann, $O$. ENSV TA Toim. Füüs. Matem., 27, № 3, 251-258 (1978).

7. Schreiber, $R$. In: Computing Methods in Applied Sciences and Engineering, VI (Eds R. Glowinski, J.-L. Lions). Elsevier Science Publishers B. V, (NorthHolland), INRIA, 1984, 285-295.

Ннститут кибернетики Академии наук Эстонской ССР
Поступила в редакцию 27/VI 1986

\section{O. VAARMANN}

\section{ULDISTATUD PSEUDOPOORDOPERAATORI APROKSIMEERIMISEL BASEERUVATEST MEETODITEST}

Mittelineaarse operaatorvõrrandi üldistatud lahendi arvutamiseks on vaadeldud üht iteratsioonimeetodite klassi, mis erijuhul sisaldab endas Ben-Israeli, Gauss-Newtoni ja Levenberg-Marquardti meetodid, aga ka üldistatud pseudopöördoperaatori järkjärgulisel aproksimeerimisel põhinevad meetodid. Iteratsioonimeetodite koonduvuspiirkonna laiendamiseks on kasutusele võetud iteratsioonisammu pikkust reguleerivad nn. relaksatsiooniparameetrid $\mathrm{ja}$ toodud tingimused nende parameetrite, lubatavate väärtuste hulga kohta.

\section{O. VAARMANN}

\section{OBER ITERATIONSVERFAHREN MIT APPROXIMATION DES VERALLGEMEINERTEN PSEUDOINVERSEN OPERATORS}

Es wird die Aufgabe der genäherten Lösung von nichtlinearen Operatorgleichungen in den Hilberträumen betrachtet. Für die Lösung einer Operatorgleichung im Sinne der kleinsten Quadrate wird eine allgemeine Klasse von Iterationsverfahren theoretisch untersucht, welche die Verfahren von Ben-Israel, Gauss-Newton und LevenbergMarquardt, aber auch die Iterationsverfahren mit sukzessiver Approximation des verallgemeinerten pseudoinversen Operators als wesentliche Sonderfälle enthält. Zur Konvergenzverbesserung dieser Iterationsverfahren wird ein reeller. Parameter für die Bestimmung der Iterationsschrittweite benutzt. 